\title{
MANAGEMENT OF DESTRUCTIVE AORTIC VALVE ENDOCARDITIS Ongoing search for the ideal valve substitute
}

Igor Vendramin ${ }^{1}$, Andrea Lechiancole ${ }^{1}$, Uberto Bortolotti ${ }^{1}$, and Ugolino Livi ${ }^{1}$

${ }^{1}$ University Hospital of Udine

October 7, 2020

\section{Hosted file}

Letter- JCS.pdf available at https://authorea.com/users/365252/articles/485432-management-ofdestructive-aortic-valve-endocarditis-ongoing-search-for-the-ideal-valve-substitute 